Journal of Mathematical Sciences, Vol. 129, No. 1, 2005

\title{
ON THE LAGRANGE PROBLEM ON THE OPTIMAL FORM FOR CIRCULAR COLUMNS
}

\author{
Yu. V. Egorov
}

UDC 517.958:539.3

\begin{abstract}
A new approach to the classical Lagrange problem on the form of the strongest circular column of fixed volume and height clamped at both ends is proposed. The existence of the optimal column is proved and an algorithm for finding its design is given.
\end{abstract}

CONTENTS

1. Introduction . . . . . . . . . . . . . . . . . . . . . . . 3573

2. Auxiliary Constructions _. . . . . . . . . . . . . . . . . . . 3577

3. Proof of Theorem $1 \ldots \ldots \ldots \ldots \ldots$

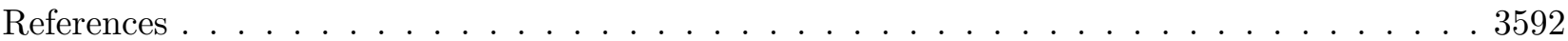

\section{Introduction}

The problem of design of the strongest elastic column of fixed volume with pinned ends was stated by Lagrange in 1773 on the basis of some works of Euler and Bernoulli (see [7]). This problem was solved in [1] by Clausen.

The recent interest in this problem was initiated by Keller and Tadjbakhsh [5, 6]. They found the optimal columns with clamped-free and clamped-hinged ends. They also obtained a unimodal optimal solution for the clamped-clamped case, but Olhoff and Rasmussen showed numerically that it is wrong. An analytical proof of this fact was given by Seyranian [11].

Despite numerous efforts and publications (see $[2,3,8,10-12]$ and the references therein), the existence of the optimal column has not yet been proved, although in 1977 Olhoff and Rasmussen found the bimodal optimal numerical solution (see [9]). Their results were later confirmed by Seyranian [10, 12] and Masur [8] by using different numerical methods.

In this paper, we propose a new approach leading to the existence theorem and give a new algorithm for finding this optimal form. Note that the equations for the function $r$ in Theorem 1 below are formally close to those found by Seyranian and Gura [10,12] and Masur [8], but the meaning of these equations here is very different; they come from solving an auxiliary problem of minimizing a nonlinear functional. We study the stationary points of this functional and show that one of them is connected with the Lagrange problem. Theorem 1 can serve as a mathematical base for some results obtained earlier by Olhoff and Rasmussen, Seyranian, and Masur. The proposed method can also be applied to the study of columns with elastic ends considered in [11]. Our approach allows one to study other similar unsolved problems, for example, the problem on the optimal form of circular columns with thin walls.

Now we state the problem using the Pearson formulation. Consider a vertical column whose horizontal sections are disks centered at the points of the $x$-axis for $0 \leq x \leq h$. (Actually, the horizontal sections can have other forms; they must be homothetic to a fixed plane figure with centers of homotheties lying on the $x$-axis.) Let $V$ be the volume of the column and $E$ be the Young modulus of it. If $z(x)$ is the

Translated from Sovremennaya Matematika i Ee Prilozheniya (Contemporary Mathematics and Its Applications), Vol. 10, Suzdal Conference-4, 2003. 
lateral deflection of the column at a point $x$, then the potential energy is

$$
T=\int_{0}^{h} E I(x) z^{\prime \prime}(x)^{2} d x-\lambda \int_{0}^{h} z^{\prime}(x)^{2} d x,
$$

where $I(x)$ is the second moment of area of the cross section of the column and $\lambda$ is the magnitude of the axial load. We neglect the weight of the column. Since $z(0)=0$ and $z^{\prime}(0)=0$, the potential energy is positive for small values of $\lambda$ for all $z \neq 0$. The buckling load $\lambda_{0}$ of the column is the supremum of the values $\lambda$ such that $T \geq 0$ for any $z$. The problem considered is to find the form of the column, i.e., the function $I(x)$ for which the value $\lambda_{0}$ is maximal and

$$
\int_{0}^{h} I(x)^{1 / 2} d x=V
$$

After rescaling and the passage from $z$ to $y=z^{\prime}$, the mathematical problem takes the following form.

Problem L. Find a positive function $Q(x) \in C[0,1]$ such that

$$
\int_{0}^{1} Q(x)^{1 / 2} d x=1
$$

and for which the minimal value $\lambda$ of the functional

$$
L_{1}[Q, y] \equiv \frac{\int_{0}^{1} Q(x) y^{\prime}(x)^{2} d x}{\int_{0}^{1} y^{2}(x) d x}
$$

in the class of functions $y \in C^{1}(0,1)$ satisfying the conditions

$$
y(0)=0, \quad y(1)=0, \quad \int_{0}^{1} y(x) d x=0
$$

is maximal.

We propose a new approach based on two-dimensional variation of the functional $L_{1}$, which allows one to prove the existence of solutions and to find the optimal form of the column using the properties of the related nonlinear functional. We also propose an algorithm allowing one to find the shape of the optimal column.

Note that Cox and Overton proved in [3] the existence theorem under the supplementary conditions $0<a \leq Q(x) \leq b<\infty$. This does not imply the existence theorem for the Lagrange problem.

We begin with some heuristic considerations, which will not be used later. First, we assume that the optimal function $Q$ is symmetric, i.e., $Q(x)=Q(1-x)$. It is rather natural, and later we shall show that it is true. If $y_{0}(x)$ is an optimal function giving the minimal value to $L_{1}$, then the function $y_{0}(1-x)$ is also optimal. The Euler-Lagrange equation has the form

$$
\left(Q y^{\prime}\right)^{\prime}+\lambda y=C, \quad y(0)=y(1)=0, \quad \int_{0}^{1} y(x) d x=0 .
$$

The functions $y_{0}(x)$ and $y_{0}(1-x)$ satisfy (4). Moreover, the odd and even parts of $y_{0}$ with respect to the point $x=1 / 2$ do so also:

$$
\left(Q u^{\prime}\right)^{\prime}+\lambda u=0, \quad\left(Q v^{\prime}\right)^{\prime}+\lambda v=C,
$$


where

$$
u(x)=\left(y_{0}(x)-y_{0}(1-x)\right) / 2, \quad v(x)=\left(y_{0}(x)+y_{0}(1-x)\right) / 2,
$$

and

$$
u(0)=u(1)=0, \quad v(0)=v(1)=0, \quad \int_{0}^{1} u(x) d x=\int_{0}^{1} v(x) d x=0 .
$$

We can consider the boundary-value problem on the half-interval $[0,1 / 2]$ and use the boundary conditions of the form

$$
u(0)=u(1 / 2)=0, \quad v(0)=0, \quad \int_{0}^{1 / 2} v(x) d x=0 .
$$

Notation. $\mathcal{A}$ is the set of positive continuous functions $Q$ satisfying (1).

$S$ is the set of functions $y$ of class $C^{1}(0,1)$ satisfying $(3)$.

$S_{\text {odd }}$ and $S_{\text {even }}$ are the sets of odd and even (about $x=1 / 2$ ) functions of class $S$.

$S_{1}$ is the set of functions $u$ of class $S_{\text {odd }}$, which are positive in ]0,1/2[ and whose derivatives monotonically decrease on $[0,1 / 2]$.

$S_{2}$ is the set of functions $v$ of class $S_{\text {even }}$, which are positive on some interval $0<x<t_{p}$, negative if $t_{p}<x<1 / 2$, and whose derivatives $v^{\prime}$ monotonically decrease on $\left[0, t_{p}^{\prime}\right]$ and increase on $\left[t_{p}^{\prime}, 1 / 2\right]$.

$S_{0}$ is the set of pairs of functions $u, v$ of class $S$ such that

$$
u^{\prime}(0)>0, \quad v^{\prime}(0)>0, \quad u^{\prime}(1)>0, \quad v^{\prime}(1)<0,
$$

the function $\theta(x)=\arctan \left(v^{\prime}(x) / u^{\prime}(x)\right)$ monotonically decreases, $\theta(0)-\theta(1) \leq 3 \pi$, and the function $r(x)=u^{\prime}(x)^{2}+v^{\prime}(x)^{2}$ has in ]0, 1 [ no more than three extremum points and is monotone on the intervals between these points and the points $x=0$ and $x=1$.

We have

$$
\lambda=\frac{\int_{0}^{1 / 2} Q(x) u^{\prime}(x)^{2} d x}{\int_{0}^{1 / 2} u(x)^{2} d x}=\frac{\int_{0}^{1 / 2} Q(x) v^{\prime}(x)^{2} d x}{\int_{0}^{1 / 2} v(x)^{2} d x}=\frac{\int_{0}^{1 / 2} Q(x)\left(u^{\prime}(x)^{2}+v^{\prime}(x)^{2}\right) d x}{\int_{0}^{1 / 2}\left(u(x)^{2}+v(x)^{2}\right) d x} .
$$

This leads to the following problem, equivalent to Problem L, as will be shown later.

Problem L1. Find a positive function $Q \in \mathcal{A}$ such that the functional

$$
\lambda(Q)=\inf _{\substack{u \in S_{\text {odd }} \\ v \in S_{\text {even }}}} L[Q, u, v], \quad L[Q, u, v] \equiv \frac{\int_{0}^{1} Q(x)\left(u^{\prime}(x)^{2}+v^{\prime}(x)^{2}\right) d x}{\int_{0}^{1}\left(u(x)^{2}+v(x)^{2}\right) d x},
$$

takes its maximal value.

We set

$$
M=\max _{Q \in \mathcal{A}} \lambda(Q)=\max _{Q \in \mathcal{A}} \min _{\substack{u \in S_{\text {odd }} \\ v \in S_{\text {even }}}} L[Q, u, v] .
$$

If we change the order of max and min in this equality (which is not legitimate in general), we can easily verify that $\max _{Q \in \mathcal{A}} L[Q, u, v]$ with fixed $u$ and $v$ is attained if

$$
Q(x)=\left(u^{\prime}(x)^{2}+v^{\prime}(x)^{2}\right)^{-2} .
$$


Using the equalities

$$
\int_{0}^{1}\left(u^{\prime}(x)^{2}+v^{\prime}(x)^{2}\right)^{-1} d x=\int_{0}^{1} Q(x)^{1 / 2} d x=1,
$$

we obtain a related problem: find the extremal values of the functional

$$
F[u, v] \equiv \int_{0}^{1}\left(u^{\prime}(x)^{2}+v^{\prime}(x)^{2}\right)^{-1} d x \int_{0}^{1}\left(u(x)^{2}+v(x)^{2}\right) d x \quad u \in S_{\text {odd }}, \quad v \in S_{\text {even }}
$$

It is easy to see that

$$
F[k u, k v]=F[u, v] \quad \text { for all } k \neq 0,
$$

the minimal value of this functional is 0 , and the maximal value is $\infty$. Thus, this problem is ill-posed at least if we do not set some supplementary restrictions on $u$ and $v$.

The principal idea of our method is to find the minimal value of the functional $F[u, v]$ in $S_{1} \times S_{2}$ and to show that the optimal functions $u$ and $v$ define, by (6), the optimal shape of the most solid column. The functional $F$ has an infinite set of points of local minimum $\left(u_{k}, v_{k}\right)$ in the space $S \times S$ and $F\left[u_{k}, v_{k}\right] \rightarrow 0$ as $k \rightarrow \infty$. The first point $\left(u_{1}, v_{1}\right)$ such that $F\left[u_{1}, v_{1}\right]=\max F\left[u_{k}, v_{k}\right]$ is the most interesting to us. However, the other points are also interesting for the Lagrange problem. These points are characterized by the number of rotations of the vector $\left(u_{k}^{\prime}(t), v_{k}^{\prime}(t)\right)$ when $t$ increases from 0 to 1 .

Our main result is as follows.

Theorem 1. There exists a solution of Problem L. The optimal function $Q_{0}$ can be found from the relation

$$
Q_{0}(x)=\left(u^{\prime}(x)^{2}+v^{\prime}(x)^{2}\right)^{-2},
$$

where $u(x)$ and $v(x)$ are linearly independent solutions of the equations

$$
\begin{aligned}
& m\left(\frac{u^{\prime}(x)}{\left(u^{\prime}(x)^{2}+v^{\prime}(x)^{2}\right)^{2}}\right)^{\prime}+u=0, \quad u(0)=u(1)=0, \quad \int_{0}^{1} u(x) d x=0, \\
& m\left(\frac{v^{\prime}(x)}{\left(u^{\prime}(x)^{2}+v^{\prime}(x)^{2}\right)^{2}}\right)^{\prime}+v=C, \quad v(0)=v(1)=0, \quad \int_{0}^{1} v(x) d x=0, \\
& u(x)=-u(1-x), \quad v(x)=v(1-x) .
\end{aligned}
$$

The function $Q_{0}$ is symmetric, $Q_{0}(x)=Q_{0}(1-x)$, and can also be found as

$$
Q_{0}(x)=\frac{r^{2}(x)}{9 m^{2}}
$$

where $r$ is the solution of the problem

$$
r^{4}(x) r^{\prime}(x)^{2}=12 m\left[c_{1} r(x)^{3}-r(x)^{4}-c_{2}(3 m)^{3}\right], \quad r(0)=4 m,
$$

which is not constant on any subinterval, and

$$
c_{1}=4 m+\left(\frac{32}{9} m k\right)^{2}, \quad c_{2}=\left(\frac{512}{81} m k\right)^{2} k^{2}\left(\frac{3}{4}-k^{2}\right), \quad k=v^{\prime}(0) .
$$


If $P(r)=\left(c_{1}-r\right) r^{3}-c_{2}(3 m)^{3}$ and $r_{1}$ and $r_{2}$ are real roots of $P$, then $0<r_{1}<4 m<r_{2}<c_{1}$ and

$$
\begin{gathered}
2 \int_{r_{1}}^{4 m} \frac{r^{2} d r}{\sqrt{P(r)}}+\int_{4 m}^{r_{2}} \frac{r^{2} d r}{\sqrt{P(r)}}=\sqrt{3 m} \\
2 \int_{r_{1}}^{4 m} \frac{d r}{\left(c_{1}-r\right) \sqrt{P(r)}}+\int_{4 m}^{r_{2}} \frac{d r}{\left(c_{1}-r\right) \sqrt{P(r)}}=\frac{2 \pi}{c_{2} 3 m \sqrt{3 m}} .
\end{gathered}
$$

The latter system of two equations for $k$ and $m$ (as well as system (7), (8)) can be solved numerically. Similar equations were found earlier by Seyranian and Gura [10, 12] and Masur [8], but the content of these equations here is very different, as is shown below.

The calculation shows that

$$
m=0.019100, \quad 0.0519 \leq Q(t) \leq 1.7824, \quad k=0.27321 .
$$

The critical load $M=1 / m=52.3562$ is close to the value found in [9] by Olhoff and Rasmussen, but our calculations are simpler.

The optimal column with circular sections is formed by rotation of the curve

$$
R(x)=\frac{Q_{0}(x)^{1 / 4}}{\sqrt{\pi}}=\sqrt{\frac{r(x)}{3 \pi m}} .
$$

We have $R(0)=R(1)=0.65140$. The minimal value of $R$ is 0.2694 ; it is attained at $x=0.2449$ and $x=0.7551$. The maximal value of $R$ is 0.6519 ; it is attained at $x=1 / 2$.

Remark. The case of a clamped-hinged column where (3) is replaced by the conditions

$$
y(0)=0, \quad Q(1) y^{\prime}(1)=0, \quad \int_{0}^{1} y(x) d x=0
$$

can be studied similarly. In this case, $c_{2}=0$ and $Q_{0}(x)=r^{2}(x) / 9 m^{2}$, where $r$ is the solution of the problem

$$
r^{\prime}(x)^{2}=12 m\left(\frac{c_{1}}{r(x)}-1\right), \quad r(0)=4 m,
$$

which can be solved explicitly and gives the formulas obtained by Keller and Tadjbakhsh [6].

\section{Auxiliary Constructions}

A. We consider the following auxiliary problem. Let $\varepsilon$ be a positive number.

Problem $\mathbf{F}_{\varepsilon}$. Find the minimal value of the functional

$$
\left.F_{\varepsilon}[u, v]=\int_{0}^{1}\left[u(x)^{2}+v(x)^{2}+\varepsilon\left(u^{\prime}(x)^{2}+v^{\prime}(x)^{2}\right)\right] d x \int_{0}^{1}\left[u^{\prime}(x)^{2}+v^{\prime}(x)^{2}\right)\right]^{-1} d x
$$

in the class $S_{0} \times S_{0}$.

Lemma 1. There exist two functions $u_{0}, v_{0} \in S_{0} \times S_{0}$ such that

$$
m_{\varepsilon} \equiv \inf _{\substack{u \in S_{0} \\ v \in S_{0}}} F_{\varepsilon}[u, v]=F_{\varepsilon}\left[u_{0}, v_{0}\right] .
$$


Proof. Let $u_{k}, v_{k} \in S_{0} \times S_{0}$ be such that

$$
\int_{0}^{1}\left[u_{k}^{\prime}(x)^{2}+v_{k}^{\prime}(x)^{2}\right]^{-1} d x=1, \quad \int_{0}^{1}\left[\left(u_{k}(x)^{2}+v_{k}(x)^{2}\right)+\varepsilon\left(u_{k}^{\prime}(x)^{2}+v_{k}^{\prime}(x)^{2}\right)\right] d x \rightarrow m_{\varepsilon} .
$$

We can assume that

$$
\int_{0}^{1}\left[\left(u_{k}(x)^{2}+v_{k}(x)^{2}\right)+\varepsilon\left(u_{k}^{\prime}(x)^{2}+v_{k}^{\prime}(x)^{2}\right)\right] d x \leq m_{\varepsilon}+\varepsilon .
$$

Since

$$
\int_{0}^{1}\left[u_{k}^{\prime}(x)^{2}+v_{k}^{\prime}(x)^{2}\right] d x \leq \frac{m_{\varepsilon}}{\varepsilon}+1
$$

there exist subsequences $u_{n_{k}}$ and $v_{n_{k}}$ uniformly converging in $I$ to $u_{0}$ and $v_{0}$ such that $u_{n_{k}}^{\prime}$ and $v_{n_{k}}^{\prime}$ weakly converge in $L_{2}(I)$ to $u_{0}^{\prime}$ and $v_{0}^{\prime}$. The function $r_{k}(x)=u_{k}^{\prime}(x)^{2}+v_{k}^{\prime}(x)^{2}$ is monotone when $a_{j, k}<x<a_{j+1, k}$, where $0=a_{0, k}<a_{1, k}<\cdots<a_{L, k}=1$ and $L \leq 4$. We can assume that $a_{j, k} \rightarrow a_{j}$ as $k \rightarrow \infty$. Let $\delta>0$ and $I_{\delta}$ be the set of the points of $I=[0,1]$ such that $\left|x-a_{j}\right|>\delta$. If $x \in I_{\delta}$ and $1-8 \delta>1 / 2$, then

$$
\delta \leq u_{k}^{\prime}(x)^{2}+v_{k}^{\prime}(x)^{2} \leq 2 \frac{m_{\varepsilon}+\varepsilon}{\varepsilon} .
$$

The functions $\theta_{k}(x)=\arctan \left(v_{k}^{\prime}(x) / u_{k}^{\prime}(x)\right)$ are monotone, $0 \leq \theta_{k}(0) \leq \pi / 2,-2 \pi-\pi / 2 \leq \theta_{k}(1) \leq-2 \pi$. By the Helly theorem, there exists a subsequence $n_{k} \rightarrow \infty$ such that $r_{n_{k}}(x)$ and $\theta_{n_{k}}(x)$ converge everywhere in $I_{\delta}$ to $r_{0}(x)$ and $\theta_{0}(x)$.

Since $u_{k}^{\prime}(x)=\sqrt{r_{k}(x)} \cos \theta_{k}(x)$ and $v_{k}^{\prime}(x)=\sqrt{r_{k}(x)} \sin \theta_{k}(x)$, the sequences $u_{n_{k}}^{\prime}$ and $v_{n_{k}}^{\prime}$ converge in $I_{\delta}$ to $u_{0}^{\prime}$ and $v_{0}^{\prime}$ and the sequences $u_{n_{k}}$ and $v_{n_{k}}^{\prime}$ converge in $I_{\delta}$ to $u_{0}$ and $v_{0}$.

Let $\delta \rightarrow 0$; using the diagonalization procedure, we obtain that the functions $u_{0}$ and $v_{0}$ belong to $S_{0}$ and

$$
\int_{0}^{1}\left(\left[u_{0}^{\prime}(x)^{2}+v_{0}^{\prime}(x)^{2}\right]^{-1} d x \leq 1, \quad \int_{0}^{1}\left[u_{0}(x)^{2}+v_{0}(x)^{2}+\varepsilon\left(u_{0}^{\prime}(x)^{2}+v_{0}^{\prime}(x)^{2}\right)\right] d x \leq m_{\varepsilon} .\right.
$$

Since $m_{\varepsilon}$ is the minimal value and is positive, we indeed have the equalities. The proof is complete.

B. Let us analyze the solution obtained.

Let us show that $m_{\varepsilon} \geq \mu$, where $\mu>0$ is independent of $\varepsilon$. Indeed, assume that for some sequence $\varepsilon_{k} \rightarrow 0$, we have $m_{\varepsilon_{k}} \rightarrow 0$. Each of the functions $u_{\varepsilon}^{\prime}$ and $v_{\varepsilon}^{\prime}$ has at most three zeros in $I$. Therefore, for any $\varepsilon>0$, there exists an interval $I_{\varepsilon}=[a, b]$ of length $b-a=1 / 12$, where both $u_{\varepsilon}$ and $v_{\varepsilon}$ are monotone. We set $a^{\prime}=a+1 / 48$ and $a^{\prime \prime}=(a+b) / 2=a+1 / 24$. The inequality

$$
\int_{0}^{1}\left(u_{\varepsilon}(x)^{2}+v_{\varepsilon}(x)^{2}\right) d x \leq m_{\varepsilon}
$$

implies that there exists a point $\theta \in\left[a, a^{\prime}\right]$ such that

$$
u_{\varepsilon}(\theta)^{2}+v_{\varepsilon}(\theta)^{2} \leq 24 \sqrt{m_{\varepsilon}}, \quad\left|u_{\varepsilon}(\theta)\right|+\left|v_{\varepsilon}(\theta)\right| \leq \sqrt{48 m_{\varepsilon}} .
$$

Since

$$
\int_{a}^{b}\left(\left|u_{\varepsilon}(x)\right|+\left|v_{\varepsilon}(x)\right|\right) d x \leq \sqrt{2 m_{\varepsilon}}
$$


we have

$$
\begin{aligned}
& \int_{\theta}^{b}(b-x)\left(\left|u_{\varepsilon}^{\prime}(x)\right|+\left|v_{\varepsilon}^{\prime}(x)\right|\right) d x \leq(b-\theta)\left(\left|u_{\varepsilon}(\theta)\right|+\left|v_{\varepsilon}(\theta)\right|\right. \\
& +\int_{\theta}^{b}\left(\left|u_{\varepsilon}(x)\right|+\left|v_{\varepsilon}(x)\right|\right) d x \leq\left(\frac{\sqrt{48}}{12}+\sqrt{2}\right) \sqrt{m_{\varepsilon}} \leq 3 \sqrt{m_{\varepsilon}}
\end{aligned}
$$

and

$$
\int_{a^{\prime}}^{a^{\prime \prime}}\left(\left|u_{\varepsilon}^{\prime}(x)\right|+\left|v_{\varepsilon}^{\prime}(x)\right|\right) d x \leq 72 \sqrt{m_{\varepsilon}}=w_{\varepsilon} .
$$

The set $\left\{x ; a^{\prime}<x<a^{\prime \prime},\left|u_{\varepsilon}^{\prime}(x)\right|>\sqrt{w_{\varepsilon}}\right\}$ has measure $\leq \sqrt{w_{\varepsilon}}$. Therefore, the inequality $\left|u_{\varepsilon}^{\prime}(x)\right| \leq \sqrt{w_{\varepsilon}}$ holds on a set of measure $\geq 1 / 48-\sqrt{w_{\varepsilon}}$. The same is true for $v_{\varepsilon}^{\prime}(x)$. Therefore, on a subset of measure $1 / 48-2 \sqrt{w_{\varepsilon}}$, we have

But then

$$
u_{\varepsilon}^{\prime}(x)^{2}+v_{\varepsilon}^{\prime}(x)^{2} \leq 2 w_{\varepsilon} .
$$

$$
1=\int_{0}^{1} \frac{d x}{\left[u_{\varepsilon}^{\prime}(x)^{2}+v_{\varepsilon}^{\prime}(x)^{2}\right]} \geq \frac{1 / 48-2 \sqrt{w_{\varepsilon}}}{\left(2 w_{\varepsilon}\right)},
$$

which is impossible since $w_{\varepsilon} \rightarrow 0$ if $\varepsilon \rightarrow 0$. Hence, $m_{\varepsilon} \geq \mu>0$.

C. If in some subinterval $[a, b]$ of $I$, the functions $r_{0}$ and $\theta_{0}$ are strictly monotone, then

$$
\frac{d}{d \delta} F_{\varepsilon}\left[u_{0}+\delta \varphi, v_{0}+\delta \psi\right]=0
$$

for $\delta=0$ and any $\varphi, \psi \in S_{0} \times S_{0}$, with their supports in $[a, b]$, i.e., $u_{0}$ and $v_{0}$ satisfy the Euler-Lagrange equations

$$
m_{\varepsilon} \frac{d}{d x}\left(Q(x) u_{0}^{\prime}\right)+u_{0}=C_{1}, \quad m_{\varepsilon} \frac{d}{d x}\left(Q(x) v_{0}^{\prime}\right)+v_{0}=C_{2},
$$

where $C_{1}$ and $C_{2}$ are constants and

$$
Q(x)=-\frac{\varepsilon}{m_{\varepsilon}}+\left(u_{0}^{\prime}(x)^{2}+v_{0}^{\prime}(x)^{2}\right)^{-2} .
$$

Here $Q(x), u$, and $v$ depend on $\varepsilon$, but we drop the extra subindex. The functions $r$ and $\theta$ cannot be constant simultaneously on any subinterval $[a, b]$ of $I$. In this case, the functions $u_{0}$ and $v_{0}$ are linear. Assume that this is not true and

$$
u_{0}(x)=k x+c, \quad v_{0}(x)=k_{1} x+c_{1}, \quad a<x<b,
$$

where $k>0$. We set $\varphi(x)=u_{1}(x)-u_{0}(x)$, where $u_{1}$ is continuous,

$$
u_{1}(x)= \begin{cases}u_{0}(x) & \text { if } x<a-\delta_{1} \text { or } x>b+\delta_{2}, \\ u_{0}(x)-\delta & \text { if } a<x<b,\end{cases}
$$

where $\delta>0$ is small. If $u(x)<k x+c$ for $a-\delta_{0}<x<a$, then we set $u_{1}(x)=k x+c-\delta$ and define $\delta_{0}$ by the condition $u_{0}\left(a-\delta_{0}\right)=k\left(a-\delta_{0}\right)+c-\delta$. Of course, it is possible only if $\delta$ is sufficiently small. If $u(x)>k x+c$ for $a-\delta_{0}<x<a$, then we set $u_{1}(x)=u_{0}^{\prime}\left(a-\delta_{0}\right)\left(x-a+\delta_{0}\right)$ on this interval and define $\delta_{0}$ by the condition $u_{0}^{\prime}\left(a-\delta_{0}\right) \delta_{0}=\delta$. We apply the same construction on the interval $b<x<b+\delta_{0}$.

In order to make $u_{1}$ satisfy the condition

$$
\int_{0}^{1} u_{1}(x) d x=0
$$


we must compensate the value of the integral on some other subinterval of $[0,1]$, where $u_{0}$ satisfies the Lagrange equation, setting $u_{1}=u_{0}+\delta \varphi(x)$ with the support of $\varphi$ on this subinterval. Then $u_{1} \in S_{0}$ and

$$
F_{\varepsilon}\left[u_{1}, v_{0}\right]-F_{\varepsilon}\left[u_{0}, v_{0}\right] \leq-2(b-a) \delta-2 m_{\varepsilon} \int_{0}^{1} \frac{\varphi^{\prime}(x) u_{0}^{\prime}(x) d x}{\left(u_{0}^{\prime}(x)^{2}+v_{0}^{\prime}(x)^{2}\right)^{2}}+o(\delta)=-2(b-a) \delta+o(\delta)<0
$$

for small $\delta>0$, which contradicts to the optimality of $u_{0}, v_{0}$. The case $k<0$ is considered similarly.

If the function $r_{0}$ is monotone on $[a, b]$ but $\theta_{0}(x)=$ const on this interval, then $v_{0}^{\prime}(x)=k u_{0}^{\prime}(x)$ and $v_{0}(x)=k u_{0}(x)+c$. We can vary $u_{0}$ and $v_{0}$ in such a way that $\psi(x)=k \varphi(x)$ and obtain the equations

$$
-\varepsilon u_{0}^{\prime \prime}(x)+m\left(u_{0}^{\prime}(x)^{-3}\right)^{\prime}+u_{0}(x)=C_{1}, \quad-\varepsilon v_{0}^{\prime \prime}(x)+m\left(v_{0}^{\prime}(x)^{-3}\right)^{\prime}+v_{0}(x)=C_{2} .
$$

Then Eqs. (9) hold.

If the function $\theta_{0}$ is monotone on $[a, b]$ but $r_{0}(x)=R=$ const on this interval, then $v_{0}^{\prime}(x)=R \cos \theta_{0}(x)$, $u_{0}^{\prime}(x)=R \sin \theta_{0}(x)$, and

$$
\begin{gathered}
\left.\int_{a}^{b}\left[u_{0}(t)^{2}+v_{0}(t)^{2}+\varepsilon\left(u_{0}^{\prime}(t)^{2}+v_{0}^{\prime}(t)^{2}\right)\right)\right] d t \int_{a}^{b} \frac{d t}{\left[\left(u_{0}^{\prime}(t)^{2}+v_{0}^{\prime}(t)^{2}\right)\right]} \\
=\varepsilon(b-a)^{2}+(b-a) / R^{2} \int_{a}^{b}\left[u_{0}(t)^{2}+v_{0}(t)^{2}\right] d t
\end{gathered}
$$

Therefore, the integral $\int_{a}^{b}\left(u^{2}+v^{2}\right) d t$ takes its minimal value for $u=u_{0}$ and $v=v_{0}$. Consider a variation of $\theta$ such that $\theta_{1}(x)=\theta_{0}(x)+\delta \varphi(x)$ with small $\delta>0$ and $\varphi \in C_{0}^{\infty}(a, b)$. We have

$$
\int_{a}^{b}\left[u_{0}(t) \int_{a}^{t} \sin \theta_{0}(s) \varphi(s) d s-v_{0}(t) \int_{a}^{t} \cos \theta_{0}(s) \varphi(s) d s\right] d t=0
$$

i.e.,

$$
\int_{a}^{b}\left[\sin \theta_{0}(s) \int_{s}^{b} u_{0}(t) d t-\cos \theta_{0}(s) \int_{s}^{b} v_{0}(t) d t\right] \varphi(s) d s=0
$$

if

$$
\int_{a}^{b} \sin \theta_{0}(s) \varphi(s) d s=0, \quad \int_{a}^{b} \cos \theta_{0}(s) \varphi(s) d s=0 .
$$

Therefore,

$$
\sin \theta_{0}(s) \int_{s}^{b} u_{0}(t) d t-\cos \theta_{0}(s) \int_{s}^{b} \varphi(t) d t=c_{1} \sin \theta_{0}(s)+c_{2} \cos \theta_{0}(s) .
$$

Since $\theta_{0}^{\prime}(s)<0$, we have

$$
\int_{s}^{b} u_{0}(t) d t=c_{1}+k \cos \theta_{0}(s), \quad \int_{s}^{b} v_{0}(t) d t=c_{2}+k \sin \theta_{0}(s) .
$$

Therefore,

$$
v_{0}(s)=-k \theta_{0}^{\prime}(s) \cos \theta_{0}(s), \quad u_{0}(s)=k \theta_{0}^{\prime}(s) \sin \theta_{0}(s), \quad u_{0}(s)^{2}+v_{0}(s)^{2}=k^{2} \theta_{0}^{\prime}(s)^{2} .
$$


Thus, the integral $\int_{a}^{b} \theta^{\prime}(s)^{2} d s$ has the minimal value under the condition $\int_{a}^{b} \theta^{\prime}(s) d s=$ const if $\theta(x)=\theta_{0}(x)$. Then $\theta^{\prime}(x)=-k=$ const and $\theta_{0}(x)=\theta_{1}-k x$. Therefore,

$$
u_{0}(x)=u_{1}-k^{2} \sin \left(\theta_{1}-k x\right), \quad v_{0}(x)=v_{1}+k^{2} \cos \left(\theta_{1}-k x\right)
$$

and again Eqs. (9) hold.

Multiplying the first equation in (9) by $u_{0}^{\prime}$ and the second equation by $v_{0}^{\prime}$, then adding and integrating, we obtain

$$
-\varepsilon\left(u_{0}^{\prime}(x)^{2}+v_{0}^{\prime}(x)^{2}\right)+\frac{3 m_{\varepsilon}}{u_{0}^{\prime}(x)^{2}+v_{0}^{\prime}(x)^{2}}+\left(u_{0}-C_{1}\right)^{2}+\left(v_{0}-C_{2}\right)^{2}=C_{3}
$$

with a constant $C_{3}$. After integrating this equality over $I$, we see that

$$
C_{3}=C_{1}^{2}+C_{2}^{2}+3 m_{\varepsilon}+\int_{0}^{1}\left[u_{0}(x)^{2}+v_{0}(x)^{2}-\varepsilon\left(u_{0}^{\prime}(x)^{2}+v_{0}^{\prime}(x)^{2}\right)\right] d x\left(u_{0}^{\prime}(x)^{2}+v_{0}^{\prime}(x)^{2}\right) d x .
$$

Since

we have

$$
\varepsilon \int_{0}^{1}\left[u_{0}^{\prime}(x)^{2}+v_{0}^{\prime}(x)^{2}\right] d x=m_{\varepsilon}-\int_{0}^{1}\left(u_{0}(x)^{2}+v_{0}(x)^{2}\right) d x
$$

$$
C_{3}=C_{1}^{2}+C_{2}^{2}+2 m_{\varepsilon}+2 \int_{0}^{1}\left(u_{0}(x)^{2}+v_{0}(x)^{2}\right) d x
$$

If we set $\rho_{0}=u_{0}^{\prime}(0)^{2}+v_{0}^{\prime}(0)^{2}$, then $(10)$ implies that $-\varepsilon \rho_{0} / m_{\varepsilon}+3 / \rho_{0}<4$. Note that, by the Cauchy inequality, $m_{\varepsilon} \geq \varepsilon$. Therefore, $3 / \rho_{0}<4+\rho_{0}$ or $\rho_{0}^{2}+4 \rho_{0}>3$. Hence, $\rho_{0}>1 / 2$ and $Q(0)<4$.

Since $-\varepsilon \rho_{0} / m_{\varepsilon}+3 / \rho_{0}>2$, we see that $\rho_{0}<3 / 2$ and $Q(0)>-5 / 9$.

Moreover, $\rho_{0}=u_{0}^{\prime}(1)^{2}+v_{0}^{\prime}(1)^{2}$ and $Q(1)=Q(0)$. Integrating Eqs. (9) over $I$, we see that

$$
C_{1}=m_{\varepsilon}\left[Q(1) u_{0}^{\prime}(1)-Q(0) u_{0}^{\prime}(0)\right], \quad C_{2}=m_{\varepsilon}\left[Q(1) v_{0}^{\prime}(1)-Q(0) v_{0}^{\prime}(0)\right] .
$$

Therefore,

$$
C_{1}^{2}+C_{2}^{2} \leq 2 m_{\varepsilon}^{2} Q(1)^{2}\left[u_{0}^{\prime}(1)^{2}+v_{0}^{\prime}(1)^{2}\right]+2 m_{\varepsilon}^{2} Q(0)^{2}\left[u_{0}^{\prime}(0)^{2}+v_{0}^{\prime}(0)^{2}\right]=4 m_{\varepsilon}^{2} Q(0)^{2} \rho_{0} .
$$

We have $Q(0)^{2} \rho_{0} \leq 24$. Hence, $C_{1}^{2}+C_{2}^{2} \leq 96 m_{\varepsilon}^{2}$ and $C_{1}^{2}+C_{2}^{2}$ is uniformly bounded in $\varepsilon$ for small $\varepsilon>0$.

If we multiply the first equation in (9) by $v_{0}-C_{2}$ and the second equation by $u_{0}-C_{1}$ and subtract, we obtain after integration

$$
Q(x)\left[u_{0}^{\prime}(x)\left(v_{0}(x)-C_{2}\right)-v_{0}^{\prime}(x)\left(u_{0}(x)-C_{1}\right)\right]=C_{4}
$$

with some constant $C_{4}$,

$$
C_{4}=Q(0)\left[C_{1} v_{0}^{\prime}(0)-C_{2} u_{0}^{\prime}(0)\right]=m_{\varepsilon} Q(0)^{2}\left[u_{0}^{\prime}(1) v_{0}^{\prime}(0)-v_{0}^{\prime}(1) u_{0}^{\prime}(0)\right] .
$$

Therefore,

$$
\left|C_{4}\right| \leq 2 m_{\varepsilon} Q(0)^{2} \rho_{0} \leq 24 m_{\varepsilon}
$$

We set

$$
r(x)=u_{0}^{\prime}(x)^{2}+v_{0}^{\prime}(x)^{2}, \quad \rho(x)=\left(u_{0}(x)-C_{1}\right)^{2}+\left(v_{0}(x)-C_{2}\right)^{2} .
$$

Then

and by (10),

$$
Q(x)=-\varepsilon / m_{\varepsilon}+r(x)^{-2}=\frac{m_{\varepsilon}-\varepsilon r^{2}}{m_{\varepsilon} r^{2}}
$$

$$
-\varepsilon r(x)+3 m_{\varepsilon} / r(x)+\rho(x)=C_{3},
$$


i.e.,

$$
r(x)=\frac{6 m_{\varepsilon}}{C_{3}-\rho(x)+\sqrt{\left(C_{3}-\rho(x)\right)^{2}+12 m_{\varepsilon} \varepsilon}} .
$$

Solving the system

$$
\begin{gathered}
u_{0}^{\prime}(x)^{2}+v_{0}^{\prime}(x)^{2}=r(x), \\
u_{0}^{\prime}(x)\left(v_{0}(x)-C_{2}\right)-v_{0}^{\prime}(x)\left(u_{0}(x)-C_{1}\right)=\rho_{1}(x),
\end{gathered}
$$

where

$$
\begin{gathered}
\rho_{1}(x)=\frac{C_{4}}{Q(x)}=\frac{C_{4} m_{\varepsilon} r(x)^{2}}{m_{\varepsilon}-\varepsilon r(x)^{2}}=\frac{36 C_{4} m_{\varepsilon}^{2}}{\left(C_{3}-\rho+R\right)^{2}-36 m_{\varepsilon} \varepsilon} \\
R(x)=\sqrt{\left(C_{3}-\rho(x)\right)^{2}+12 m_{\varepsilon} \varepsilon},
\end{gathered}
$$

we obtain

$$
\begin{gathered}
u_{0}^{\prime}(x)=\frac{\rho_{1}(x)}{\rho(x)}\left(v_{0}(x)-C_{2}\right) \pm \frac{\rho_{2}(x)}{\rho(x)}\left(u_{0}(x)-C_{1}\right), \\
v_{0}^{\prime}(x)=-\frac{\rho_{1}(x)}{\rho(x)}\left(u_{0}(x)-C_{1}\right) \pm \frac{\rho_{2}(x)}{\rho(x)}\left(v_{0}(x)-C_{2}\right),
\end{gathered}
$$

where

$$
\rho_{2}(x)=\sqrt{\rho(x) r(x)-\rho_{1}(x)^{2}} .
$$

This implies that

$$
\left(u_{0}(x)-C_{1}\right) u_{0}^{\prime}(x)+\left(v_{0}(x)-C_{2}\right) v_{0}^{\prime}(x)= \pm \sqrt{\rho(x) r(x)-\rho_{1}(x)^{2}} .
$$

Therefore,

$$
\rho^{\prime}(x)^{2}=4 \rho_{2}(x)^{2}=G_{\varepsilon}(\rho(x)),
$$

where

$$
G_{\varepsilon}(\rho)=4 \frac{6 \rho\left(\left[\left(C_{3}-\rho+R\right)^{2}-36 m_{\varepsilon} \varepsilon\right]^{2}-\left(36 m_{\varepsilon}^{2} C_{4}\right)^{2}\left(C_{3}-\rho+R\right)\right.}{\left(C_{3}-\rho+R\right)\left(\left[\left(C_{3}-\rho+R\right)^{2}-36 m_{\varepsilon} \varepsilon\right]^{2}\right.} .
$$

Moreover,

$$
\rho(0)=\rho(1)=C_{1}^{2}+C_{2}^{2} .
$$

Since $\rho(0)<3 / 2$, we see that $Q(0)>4 / 9-\varepsilon / m_{\varepsilon}>1 / 3$ for small $\varepsilon>0$.

D. Let us show that the function $Q$ is symmetric, i.e., $Q(x)=Q(1-x)$. As we showed above, $Q(0)=Q(1)$ and

$$
u_{0}^{\prime}(1)=u_{0}^{\prime}(0)+C_{1} / Q(0) m_{\varepsilon}, \quad v_{0}^{\prime}(1)=v_{0}^{\prime}(0)+C_{2} / Q(0) m_{\varepsilon} .
$$

Therefore,

$$
\begin{gathered}
\rho^{\prime}(1)=2\left(u_{0}(1)-C_{1}\right) u_{0}^{\prime}(1)+2\left(v_{0}(1)-C_{2}\right) v_{0}^{\prime}(1)=-2\left(C_{1} u_{0}^{\prime}(1)+C_{2} v_{0}^{\prime}(1)\right) \\
=\rho^{\prime}(0)-2\left(C_{1}^{2}+C_{2}^{2}\right) / Q(0) m_{\varepsilon}<\rho^{\prime}(0) .
\end{gathered}
$$

Since $\rho^{\prime}(1)= \pm \rho^{\prime}(0)$ by (14), this means that $\rho^{\prime}(1)=-\rho^{\prime}(0)$. Therefore, (14) implies that the function $r_{\varepsilon}(x)$ is even and the function $Q_{\varepsilon}$ is also even.

Now we show that the minimal value of the functional $F_{\varepsilon}$ in $S_{1} \times S_{2}$ coincides with $m_{\varepsilon}$. It suffices to verify that one of the functions $u_{0}$ and $v_{0}$ is odd and the other is even.

Let us show that $u_{0} \not \equiv 0$. Indeed, if $F_{\varepsilon}\left[0, v_{0}\right]=m_{\varepsilon}$, then

$$
\frac{d}{d \delta} F_{\varepsilon}\left[\sqrt{\delta} \varphi, v_{0}\right]=\int_{0}^{1}\left(\varphi(x)^{2}+\varepsilon \varphi^{\prime}(x)^{2}\right) d x-m_{\varepsilon} \int_{0}^{1} \frac{\varphi^{\prime}(x)^{2}}{v_{0}^{\prime}(x)^{4}} d x
$$


for $\delta=0$. Let $[a, b]$ be a subinterval of $I$ where $v_{0}^{\prime}(x)^{4} \leq c$. We set $b-a=4 l$,

$$
\varphi(x)= \begin{cases}x-a, & x \in(a, a+l), \\ -x+a+2 l, & x \in(a+l, a+3 l), \\ \varphi(x)=x-b, & x \in(a+3 l, b) .\end{cases}
$$

Then

$$
\int_{0}^{1} \varphi(x) d x=0, \quad \int_{0}^{1} \frac{\varphi^{\prime}(x)^{2}}{v_{0}^{\prime}(x)^{4}} d x \geq \frac{4 l}{c} .
$$

We can take $l$ and $\varepsilon$ sufficiently small such that

$$
\int_{a}^{b}\left(\varphi^{2}+\varepsilon \varphi^{\prime}(x)^{2}\right) d x=4 \int_{a}^{a+l}(x-a)^{2} d x+\varepsilon l=4\left(\frac{l^{3}}{3}+\varepsilon l\right)<\frac{4 m_{\varepsilon} l}{c} .
$$

Therefore, $\frac{d}{d \delta} F_{\varepsilon}\left[\sqrt{\delta} \varphi, v_{0}\right]<0$ for $\varepsilon=0$ and $m_{\varepsilon}$ is not the minimal value of $F_{\varepsilon}$.

Similarly, we can verify that $v_{0} \not \equiv 0$. The functions $u_{0}$ and $v_{0}$ are linearly independent since the relation $u=k v$ implies the equality $F_{\varepsilon}[u, v]=F_{\varepsilon}[0, v]$.

Let us show that the functions $u_{0}$ and $v_{0}$ cannot both be odd. If this would be so, then $C_{1}=C_{2}=0$ and we can assume that $u_{0}$ and $v_{0}$ are positive in $] 0,1 / 2[$. Then by the Sturm theorem, they are linearly dependent, which is impossible, as we have shown.

For the same reason, the functions $u_{0}$ and $v_{0}$ cannot both be even. In this case, the functions $u_{0}-C_{1}$ and $v_{0}-C_{2}$ satisfy the same homogeneous equation $m\left(Q z^{\prime}\right)^{\prime}+z=0$ and their derivatives vanish at $x=1 / 2$; therefore, they are linearly dependent, i.e., $k_{1}\left(u_{0}-C_{1}\right)+k_{2}\left(v_{0}-C_{2}\right)=0$. Since $u_{0}(0)=v_{0}(0)$, this implies that $k_{1} u_{0}(x)+k_{2} v_{0}(x)=0$.

We know that

$$
u_{0}^{\prime}(0)^{2}+v_{0}^{\prime}(0)^{2}=u_{0}^{\prime}(1)^{2}+v_{0}^{\prime}(1)^{2}, \quad C_{1} v_{0}^{\prime}(0)-C_{2} u_{0}^{\prime}(0)=C_{1} v_{0}^{\prime}(1)-C_{2} u_{0}^{\prime}(1) .
$$

If $C_{1} \neq 0$ and $C_{2} \neq 0$, then either $u_{0}^{\prime}(0)=u_{0}^{\prime}(1)$ and $v_{0}^{\prime}(0)=v_{0}^{\prime}(1)$ or $u_{0}^{\prime}(0)=-u_{0}^{\prime}(1)$ and $v_{0}^{\prime}(0)=-v_{0}^{\prime}(1)$. In the first case, both $u_{0}$ and $v_{0}$ are odd, and in the second case, they are both even. Since this is impossible, one of the constants $C_{j}$ vanishes. Assume that $C_{1}=0$. Then $C_{2} \neq 0$ and $u_{0}$ is odd. Since $v_{0}^{\prime}(0)^{2}=v_{0}^{\prime}(1)^{2}$ and $v_{0}$ cannot be odd, it is even.

E. Now we consider the following problem.

Problem F. Find the functions $u$ and $v$ of class $S_{0}$ giving the minimal value of the functional

$$
F[u, v]=\int_{0}^{1}\left[u(x)^{2}+v(x)^{2}\right] d x \int_{0}^{1}\left(u^{\prime}(x)^{2}+v^{\prime}(x)^{2}\right)^{-1} d x .
$$

Lemma 2. Problem $\mathrm{F}$ has a solution $(u, v)$, which is the limit in $C^{1}(I)$ of some sequence $\left(u_{\varepsilon_{k}}, v_{\varepsilon_{k}}\right)$ of solutions of Problems $\mathrm{F}_{\varepsilon_{k}}$ as $\varepsilon_{k} \rightarrow 0$. These functions are linearly independent, belong to $C^{\infty}(0,1)$, are analytic on this interval, and satisfy Eqs. (7), (8). Moreover, we can assume that $u$ is odd and $v$ is even, so that

$$
F[u, v]=\inf _{\substack{\tilde{u} \in S_{0} \\ \tilde{v} \in S_{0}}} F[\tilde{u}, \tilde{v}]=\inf _{\substack{\tilde{u} \in S_{1} \\ \tilde{v} \in S_{2}}} F[\tilde{u}, \tilde{v}]
$$

Proof. Let $u_{\varepsilon}$ and $v_{\varepsilon}$ be the functions from $S_{1} \times S_{2}$ minimizing the functional $F_{\varepsilon}$. The function $u_{\varepsilon}$ is positive on $] 0,1 / 2\left[\right.$ and, by the Sturm theorem, $v_{\varepsilon}-C_{\varepsilon}$ has a unique zero in this interval. 
Since $C_{3} \leq 4 m_{\varepsilon}+8 m_{\varepsilon}^{2}$ and $C_{4} \leq 2 m_{\varepsilon}$, we can assume that $m_{\varepsilon} \rightarrow m, C_{\varepsilon}=C_{2 \varepsilon} \rightarrow C, C_{3 \varepsilon} \rightarrow C_{3}$, and $C_{4 \varepsilon} \rightarrow C_{4}$. From (10), we see that

$$
u_{\varepsilon}^{\prime}(0)^{2}+v_{\varepsilon}^{\prime}(0)^{2} \rightarrow \frac{3}{4}, \quad Q(0) \rightarrow \frac{16}{9} .
$$

We can assume that $v_{\varepsilon}^{\prime}(0) \rightarrow k$ and $u_{\varepsilon}^{\prime}(0) \rightarrow \sqrt{3 / 4-k^{2}}$.

As $\varepsilon \rightarrow 0$, we see from (14) that

$$
G_{\varepsilon}(\rho) \rightarrow G(\rho)=\frac{12 \rho\left(C_{3}-\rho\right)-324 m^{2} C_{4}^{2}}{\left(C_{3}-\rho\right)^{3}} .
$$

The function $G$ takes negative values at $\rho=0$ and at $\rho=C_{3}$. It has two real, positive roots, $R_{1}$ and $R_{2}$. Since $r_{\varepsilon}$ takes its values in the interval between two real roots of $G_{\varepsilon}$, we obtain that $2 R_{2} \geq r_{\varepsilon}(x) \geq R_{1} / 2$ for all small $\varepsilon$ and $0 \leq x \leq 1$.

Equalities (12) and (13) imply that the first derivatives of the functions $u_{\varepsilon}$ and $v_{\varepsilon}$ are uniformly bounded. Since

$$
\begin{aligned}
& u_{\varepsilon}^{\prime \prime}(x)\left(-\frac{\varepsilon}{m_{\varepsilon}}+\frac{3 u_{\varepsilon}^{\prime}(x)^{2}-v_{\varepsilon}^{\prime}(x)^{2}}{\left(u_{\varepsilon}^{\prime}(x)^{2}+v_{\varepsilon}^{\prime}(x)^{2}\right)^{3}}\right)+4 v_{\varepsilon}^{\prime \prime}(x) \frac{u_{\varepsilon}^{\prime}(x) v_{\varepsilon}^{\prime}(x)}{\left(u_{\varepsilon}^{\prime}(x)^{2}+v_{\varepsilon}^{\prime}(x)^{2}\right)^{3}}=\frac{C_{1}-u_{\varepsilon}(x)}{m_{\varepsilon}}, \\
& v_{\varepsilon}^{\prime \prime}(x)\left(-\frac{\varepsilon}{m_{\varepsilon}}+\frac{3 v_{\varepsilon}^{\prime}(x)^{2}-u_{\varepsilon}^{\prime}(x)^{2}}{\left(u_{\varepsilon}^{\prime}(x)^{2}+v_{\varepsilon}^{\prime}(x)^{2}\right)^{3}}\right)+4 u_{\varepsilon}^{\prime \prime}(x) \frac{u_{\varepsilon}^{\prime}(x) v_{\varepsilon}^{\prime}(x)}{\left(u_{\varepsilon}^{\prime}(x)^{2}+v_{\varepsilon}^{\prime}(x)^{2}\right)^{3}}=\frac{C_{2}-v_{\varepsilon}(x)}{m_{\varepsilon}},
\end{aligned}
$$

we have

$$
\begin{aligned}
& D_{\varepsilon}(x) u_{\varepsilon}^{\prime \prime}(x)=\left(C_{1}-u_{\varepsilon}(x)\right)\left(-\frac{\varepsilon}{m_{\varepsilon}}+\frac{3 v_{\varepsilon}^{\prime}(x)^{2}-u_{\varepsilon}^{\prime}(x)^{2}}{\left(u_{\varepsilon}^{\prime}(x)^{2}+v_{\varepsilon}^{\prime}(x)^{2}\right)^{3}}\right)-\left(C_{2}-v_{\varepsilon}(x)\right) \frac{4 u_{\varepsilon}^{\prime}(x) v_{\varepsilon}^{\prime}(x)}{\left(u_{\varepsilon}^{\prime}(x)^{2}+v_{\varepsilon}^{\prime}(x)^{2}\right)^{3}}, \\
& D_{\varepsilon}(x) v_{\varepsilon}^{\prime \prime}(x)=\left(C_{2}-v_{\varepsilon}(x)\right)\left(-\frac{\varepsilon}{m_{\varepsilon}}+\frac{3 u_{\varepsilon}^{\prime}(x)^{2}-v_{\varepsilon}^{\prime}(x)^{2}}{\left(u_{\varepsilon}^{\prime}(x)^{2}+v_{\varepsilon}^{\prime}(x)^{2}\right)^{3}}\right)-\left(C_{1}-u_{\varepsilon}(x)\right) \frac{4 u_{\varepsilon}^{\prime}(x) v_{\varepsilon}^{\prime}(x)}{\left(u_{\varepsilon}^{\prime}(x)^{2}+v_{\varepsilon}^{\prime}(x)^{2}\right)^{3}},
\end{aligned}
$$

where

$$
D_{\varepsilon}=\frac{4 \varepsilon^{2}}{3 m_{\varepsilon}}-3 m_{\varepsilon}\left(\frac{\varepsilon}{3 m_{\varepsilon}}+\frac{1}{\left(u_{\varepsilon}^{\prime}(x)^{2}+v_{\varepsilon}^{\prime}(x)^{2}\right)^{2}}\right)^{2}
$$

tends to $-3 m / \rho(x)^{4}$ as $\varepsilon \rightarrow 0$. Therefore, the second derivatives of $u_{\varepsilon}$ and $v_{\varepsilon}$ are uniformly bounded:

$$
\sup _{x}\left(\left|u_{\varepsilon}^{\prime \prime}(x)\right|+\left|v_{\varepsilon}^{\prime \prime}(x)\right|\right) \leq A .
$$

Hence the set of functions $u_{\varepsilon}$ and $v_{\varepsilon}$ is compact in $C^{1}(I)$ and we can find subsequences converging in $C^{1}(I)$ as $\varepsilon \rightarrow 0, u_{\varepsilon} \rightarrow u$, and $v_{\varepsilon} \rightarrow v$.

In particular, the last system takes, after passing to the limit, the following form:

$$
\begin{aligned}
& \frac{3 m}{\rho} u^{\prime \prime}=\left(3 v^{\prime}(x)^{2}-u^{\prime}(x)^{2}\right)\left(u-C_{1}\right)-4 u\left((x) v^{\prime} x\right)\left(v-C_{2}\right), \\
& \frac{3 m}{\rho} v^{\prime \prime}=-4 u\left((x) v^{\prime} x\right)\left(u-C_{1}\right)+\left(3 u^{\prime}(x)^{2}-v^{\prime}(x)^{2}\right)\left(v-C_{2}\right) .
\end{aligned}
$$

Now let $\mu$ be the minimum in Problem F. Since always $F[u, v] \leq F_{\varepsilon}[u, v]$, we have $\mu \leq m$. On the other hand, if $\mu<m$, then there exist $u_{1} \in S_{1}$ and $v_{1} \in S_{2}$ such that $F\left[u_{1}, v_{1}\right]<m-\delta$ with $\delta>0$. Then

$$
\left.F_{\varepsilon}\left[u_{1}, v_{1}\right]<m-\delta+\varepsilon \int_{0}^{1}\left[u_{1}^{\prime}(x)^{2}+v_{1}^{\prime}(x)^{2}\right] d x \int_{0}^{1}\left[u_{1}^{\prime}(x)^{2}+v_{1}^{\prime}(x)^{2}\right)\right]^{-1} d x<m-\frac{\delta}{2}<m_{\varepsilon}-\frac{\delta}{4}
$$

for small $\varepsilon>0$, which is impossible. Therefore, $\mu=m$ and the functions $u$ and $v$ yield a solution of Problem F. 
We set $H_{\varepsilon}(\rho)=G_{\varepsilon}\left(C_{3}-\rho\right)$. If $\varepsilon \rightarrow 0$, then $H_{\varepsilon}(\rho) \rightarrow H(\rho)=c_{1} \rho^{3}-\rho^{4}-c_{2}$, where

$$
c_{1}=4 m+\left(\frac{32}{9} m k\right)^{2}, \quad c_{2}=\left(\frac{2^{18}}{3^{5}}\right) m^{5} k^{2}\left(\frac{3}{4}-k^{2}\right)
$$

$m=\lim m_{\varepsilon}, k=v^{\prime}(0)$. Therefore, there exist positive limit values of the real roots $R_{1}$ and $R_{2}$. They serve as the bounds for the limit function $\rho(t)$. Moreover, $R_{2}<c_{1}=\lim _{\varepsilon \rightarrow 0} C_{3}$ and, by $(10), \rho(x) \geq 3 m_{\varepsilon} / b_{1}$.

In any case, we see that

$$
Q(x)=Q_{\varepsilon}(x) \rightarrow \frac{\left(c_{1}-\rho(x)\right)^{2}}{9 m^{2}} .
$$

Obviously, the function $u$ is odd and $v$ is even in [0,1]. Moreover, the function $u$ is positive in $] 0,1 / 2[$ and $v$ is positive in $] 0, t_{p}$ [ and negative in $] t_{p}, 1 / 2[$. Passing to the limit, we see that the functions $u$ and $v$ satisfy Eqs. (7) and (8).

Let us show that $u \not \equiv 0$. Indeed, if $F[0, v]=m$, then

$$
\frac{d}{d \varepsilon} F[\sqrt{\varepsilon} \varphi, v]=\int_{0}^{1} \varphi(x)^{2} d x-m \int_{0}^{1} \frac{\varphi^{\prime}(x)^{2}}{v^{\prime}(x)^{4}} d x
$$

for $\varepsilon=0$. Let $[a, b]$ be a subinterval of $I$ where $v^{\prime}(x)^{4} \leq c$. Set $b-a=4 l$,

$$
\varphi(x)= \begin{cases}x-a, & x \in(a, a+l), \\ -x+a+2 l, & x \in(a+l, a+3 l), \\ x-b, x \in(a+3 l, b) . & \end{cases}
$$

Then

$$
\int_{0}^{1} \varphi(x) d x=0
$$

We can take $l$ sufficiently small such that

$$
m \int_{0}^{1} \frac{\varphi^{\prime}(x)^{2}}{v^{\prime}(x)^{4}} d x \geq \frac{m l}{c}>4 \int_{a}^{a+l}(x-a)^{2} d x=\frac{4 l^{3}}{3},
$$

i.e., $l^{2}<3 m / 4 c$. Therefore, $\frac{d}{d \varepsilon} F[\sqrt{\varepsilon} \varphi, v]<0$ at $\varepsilon=0$ and $m$ is not the minimal value of $F$.

Similarly, we can verify that $v \not \equiv 0$. The functions $u$ and $v$ are linearly independent since the equality $u=k v$ implies that $F[u, v]=F[0, v]$.

System (7), (8) is equivalent to the following system of four first-order equations:

$$
u^{\prime}=\frac{z}{Q_{0}}, \quad z^{\prime}=-M u, \quad v^{\prime}=\frac{w}{Q_{0}}, \quad w^{\prime}=M(v-c),
$$

where $M=1 / m$ and $Q_{0}(x)=\left(u^{\prime}(x)^{2}+v^{\prime}(x)^{2}\right)^{-2}$. Since $Q_{0} \in C(I)$ and $Q_{0}>0$, we see that $u, v \in C^{1}(I)$. Therefore, $Q_{0} u^{\prime} \in C^{1}(I), Q_{0} v^{\prime} \in C^{1}(I)$, and

$$
\left(Q_{0} u^{\prime}\right)^{2}+\left(Q_{0} v^{\prime}\right)^{2}=\frac{1}{\left(u^{\prime 2}+v^{\prime 2}\right)^{2}} \in C^{1}(I) .
$$

Hence, $Q_{0} \in C^{1}(I)$ and $u, v, z, w \in C^{2}(I)$. We can continue and obtain that $u, v \in C^{\infty}(I)$. It is easy to see that the functions $u, v, z, w$, and $Q$ are analytic in $I$. 
F. We say that $u \in S_{0}(a, b)$, where $0 \leq a<b \leq 1$, if $u(a+x(b-a)) \in S_{0}$.

Lemma 3. Let $f$ be a positive function from $C(0,1)$ monotone in $\left(a_{j}, a_{j+1}\right)$, where $j=0,1,2,3$ and $0=a_{0} \leq a_{1} \leq a_{2} \leq a_{3} \leq a_{4}=1$. Then there exist two functions $u$ and $v$ from $S_{0}$ such that

$$
f=u^{\prime 2}+v^{\prime 2} \text {. }
$$

Proof. Set

$$
u(x)=\int_{0}^{x} \sqrt{f(t)} \cos \theta(t) d t, \quad v(x)=\int_{0}^{x} \sqrt{f(t)} \sin \theta(t) d t .
$$

Let $\Phi$ be the set of monotonically decreasing functions $\theta$ such that $0 \leq \theta(0) \leq \pi / 2$ and $-5 \pi / 2 \leq \theta(1) \leq$ $-2 \pi$. It is easy to verify that $(u, v) \in S_{0}$ if and only if $\theta \in \Phi$ and

$$
\begin{array}{ll}
\int_{0}^{1} \sqrt{f(t)} \cos \theta(t) d t=0, & \int_{0}^{1} \sqrt{f(t)} \sin \theta(t) d t=0, \\
\int_{0}^{1} t \sqrt{f(t)} \cos \theta(t) d t=0, & \int_{0}^{1} t \sqrt{f(t)} \sin \theta(t) d t=0 .
\end{array}
$$

We set

$$
\begin{gathered}
\alpha=\int_{0}^{1} \sqrt{f(t)} \cos \theta(t) d t, \quad \beta=\int_{0}^{1} \sqrt{f(t)} \sin \theta(t) d t \\
\gamma=\int_{0}^{1} t \sqrt{f(t)} \cos \theta(t) d t, \quad \delta=\int_{0}^{1} t \sqrt{f(t)} \sin \theta(t) d t
\end{gathered}
$$

and $B[\theta]=\alpha^{2}+\beta^{2}+\gamma^{2}+\delta^{2}$.

Let $\mu$ be the minimum of $B$ in $\Phi$ when the function $f$ is fixed. It is easy to see that the set $\Phi$ is compact by the Helly theorem. Therefore, if $B\left[\theta_{k}\right] \rightarrow 0$, we can assume that $\theta_{k}(x) \rightarrow \theta_{0}(x)$ for almost all $x$ and $B\left[\theta_{0}\right]=\mu$.

Let us show that $\mu=0$. Assume that $\theta_{0}$ is strictly monotone in a subdomain $\omega \subset[0,1]$ and $\varphi \in C_{0}(\omega)$. Then

$$
\frac{d}{d \varepsilon} B\left[\theta_{0}+\varepsilon \varphi\right]=0
$$

for $\varepsilon=0$, i.e.,

$$
\int_{0}^{1} \sqrt{f(t)}\left[-\alpha \sin \theta_{0}(t)+\beta \cos \theta_{0}(t)-\gamma t \sin \theta_{0}(t)+\delta t \cos \theta_{0}(t)\right] \varphi(t) d t=0 .
$$

Therefore,

$$
(\alpha+x \gamma) \sin \theta_{0}(x)=(\beta+x \delta) \cos \theta_{0}(x)
$$

i.e., either $\mu=0$ or

$$
\sin \theta_{0}(x)=\frac{\beta+x \delta}{r(x)}, \quad \cos \theta_{0}(x)=\frac{\alpha+x \gamma}{r(x)}, \quad r(x)^{2}=(\beta+x \delta)^{2}+(\alpha+x \gamma)^{2} .
$$

However, then $u^{\prime}$ and $v^{\prime}$ can change their signs only once, which is impossible for $\theta_{0} \in \Phi$. Therefore, $\mu=0$ and $\left(u_{0}, v_{0}\right) \in S_{0}$. The proof is complete. 
G.

Lemma 4. Let $Q$ be a continuous, positive function defined in $[0,1 / 2], Q(0)>1$. Consider the following Sturm-Liouville problem:

$$
\left(Q(x) y^{\prime}\right)^{\prime}+\lambda y=0 \quad \text { on } \quad[0,1 / 2], \quad 2 Q(0) y^{\prime}(0)=\lambda y(0), \quad y^{\prime}(1 / 2)=0 .
$$

Its spectrum is discrete, $\lambda_{j} \rightarrow \infty$ as $j \rightarrow \infty, \lambda_{0}=0<\lambda_{1}<\ldots$, and the eigenvalues are simple. For positive $j$, the number of zeros of the eigenfunction $\varphi_{j}$ corresponding to $\lambda_{j}$ is exactly $j$ and the number of zeros of $\varphi_{j}^{\prime}$ in $[0,1 / 2[$ is also $j$.

Proof. If $\lambda<0$, then

$$
-\int_{0}^{1 / 2} Q(x) y^{\prime}(x)^{2} d x+\lambda / 2 y^{2}(0)+\lambda \int_{0}^{1 / 2} y^{2}(x) d x
$$

and, therefore, $y \equiv 0$. If $\lambda=0$, then $y(x)=$ const.

Positive eigenvalues $\lambda_{j}$ can be found as

$$
\lambda_{j}=\inf _{y \in M_{j}} \int_{0}^{1 / 2} Q(x) y^{\prime}(x)^{2} d x
$$

where $M_{j}$ is the set of functions $y \in H^{1}(0,1 / 2)$ such that

$$
\int_{0}^{1 / 2} y(x)^{2} d x-y(0)^{2} / 2=1, \quad \int_{0}^{1 / 2} y(x) \varphi_{i}(x) d t=y(0) \varphi_{i}(0) / 2, \quad 0 \leq i<j .
$$

Note that for $i=0$, the last condition means that

$$
y(0)=2 \int_{0}^{1 / 2} y(x) d x
$$

and, therefore,

$$
y(0)^{2}<2 \int_{0}^{1 / 2} y(x)^{2} d x
$$

if $y(x) \not \equiv$ const. The rest of the proof is standard (see, e.g., [4, p. 166]).

Lemma 5. Let

$$
M=\sup _{Q \in \mathcal{A}} \inf _{\substack{u \in S_{0} \\ v \in S_{0}}} \frac{\int_{0}^{1} Q(x)\left(u^{\prime}(x)^{2}+v^{\prime}(x)^{2}\right) d x}{\int_{0}^{1}\left(u(x)^{2}+v(x)^{2}\right) d x} .
$$

Then $M \leq 1 / m$, where $m$ was defined in Lemma 2 .

Proof. Let $Q$ be a function from $\mathcal{A} \cap C^{2}(I)$. If $Q$ has less than five intervals of monotonicity, then we can apply Lemma 3 and find $(u, v) \in S_{0}$ such that

$$
f(x)=\frac{1}{\sqrt{Q}}=u^{\prime}(x)^{2}+v^{\prime}(x)^{2}
$$

in $I$. Since

$$
\int_{0}^{1} Q(x)^{1 / 2} d x=1
$$


we have

$$
\frac{\int_{0}^{1} Q(x)\left(u^{\prime}(x)^{2}+v^{\prime}(x)^{2}\right) d x}{\int_{0}^{1}\left(u(x)^{2}+v(x)^{2}\right) d x}=\frac{\int_{0}^{1}\left(u^{\prime}(x)^{2}+v^{\prime}(x)^{2}\right)^{-1} d x}{\int_{0}^{1}\left(u(x)^{2}+v(x)^{2}\right) d x}=\frac{1}{F[u, v]} \leq \frac{1}{m} .
$$

If $Q$ has more than four intervals of monotonicity, then we can choose a subinterval $[a, b]$ where $Q$ has exactly four intervals of monotonicity and set $u=v=0$ outside this subinterval. We can construct the functions $u$ and $v$ as above. Then

$$
\int_{a}^{b} Q(x)^{1 / 2} d x \leq 1,
$$

and the above arguments hold.

H.

Lemma 6. Let $u$ and $v$ be the functions found in Lemma 2. Let $k=v^{\prime}(0), c_{1}=4 m+(32 m k / 9)^{2}$, and $c_{2}=512 m k \sqrt{3 / 4-k^{2}}$. We set $r(x)=c_{1}-u(x)^{2}-(v(x)-C)^{2}$. Then

$$
r^{\prime}(x)^{4} r(x)^{2}=12 m\left(\left(c_{1}-r(x)\right) r(x)^{3}-c_{2}^{2}(3 m)^{3}\right) .
$$

The function $r$ cannot take a constant value on any nonempty subinterval of $I$.

Proof. Multiplying Eqs. (7) and (8) by $u^{\prime}$ and $v^{\prime}$, respectively, and adding, we obtain

$$
-3 m \frac{u^{\prime}(x) u^{\prime \prime}(x)+v^{\prime}(x) v^{\prime \prime}(x)}{\left(u^{\prime}(x)^{2}+v^{\prime}(x)^{2}\right)^{2}}+u(x) u^{\prime}(x)+(v(x)-C) v^{\prime}(x)=0 .
$$

After integration, we obtain

$$
\frac{3 m}{u^{\prime}(x)^{2}+v^{\prime}(x)^{2}}+\left[u(x)^{2}+(v(x)-C)^{2}\right]=c_{1} .
$$

Integrating this equality over $(0,1)$, we have

$$
c_{1}=4 m+C^{2} .
$$

Setting $x=0$ and using (7) and (8), we can see that

$$
u^{\prime}(0)^{2}+v^{\prime}(0)^{2}=\frac{3}{4}, \quad Q(0)=\frac{16}{9}, \quad C=-\frac{32}{9} m v^{\prime}(0) .
$$

On the other hand, multiplying Eqs. (7) and (8) by $v-C$ and $u$ and subtracting, we see that

$$
m\left(\frac{u^{\prime}(x)(v(x)-C)-v^{\prime}(x) u(x)}{\left(u^{\prime}(x)^{2}+v^{\prime}(x)^{2}\right)^{2}}\right)^{\prime}=0,
$$

i.e.,

$$
u^{\prime}(x)(v(x)-C)-v^{\prime}(x) u(x)=c_{2}\left(u^{\prime}(x)^{2}+v^{\prime}(x)^{2}\right)^{2} .
$$

Setting $x=0$, we see that

Set

$$
c_{2}=-\frac{16}{9} C u^{\prime}(0)=\frac{512}{81} m u^{\prime}(0) v^{\prime}(0) .
$$

By (15) and (16), we have

$$
\rho(x)=u(x)^{2}+(v(x)-C)^{2} .
$$

$$
\begin{gathered}
u^{\prime}(x)^{2}+v^{\prime}(x)^{2}=\frac{3 m}{c_{1}-\rho} \\
u^{\prime}(x)(v(x)-C)-v^{\prime}(x) u(x)=c_{2}\left(\frac{3 m}{c_{1}-\rho}\right)^{2} .
\end{gathered}
$$


Solving this system for $u^{\prime}(x)$ and $v^{\prime}(x)$, we obtain that

$$
\begin{gathered}
\rho(x) u^{\prime}(x)=c_{2}(v(x)-C) f(x)^{2} \pm u(x) \sqrt{f(x) \rho(x)-c_{2}^{2} f(x)^{4}} \\
\rho(x) v^{\prime}(x)=-c_{2} u(x) f(x)^{2} \pm(v(x)-C) \sqrt{f(x) \rho(x)-c_{2}^{2} f(x)^{4}}
\end{gathered}
$$

where $f(x)=3 m /\left(c_{1}-\rho(x)\right)$. Multiplying (17) by $u$ and (18) by $v(x)-C$ and summing, we find that

$$
\rho^{\prime}(x)= \pm 2 \sqrt{f(x) \rho(x)-c_{2}^{2} f(x)^{4}}, \quad \rho(0)=C^{2} .
$$

Setting $r(x)=c_{1}-\rho(x)$, we see that $r(0)=4 m$ and

$$
r^{\prime}(x)^{2}=4\left(3 m \frac{c_{1}-r(x)}{r(x)}-c_{2}^{2} \frac{(3 m)^{4}}{r(x)^{4}}\right)=\frac{12 m}{r(x)^{4}}\left(c_{1} r(x)^{3}-r(x)^{4}-c_{2}^{2}(3 m)^{3}\right) .
$$

Since $v^{\prime}(0)=k$, we have $u^{\prime}(0)=\sqrt{3 / 4-k^{2}}$,

$$
c_{1}=4 m+\left(\frac{32}{9} m k\right)^{2}, \quad c_{2}=\frac{512}{81} m k \sqrt{3 / 4-k^{2}} .
$$

The polynomial

$$
P(r)=c_{1} r^{3}-r^{4}-c_{2}^{2}(3 m)^{3}
$$

has two real roots $r_{1}$ and $r_{2}$ such that

$$
0<r_{1}<4 m<r_{2} \text {. }
$$

The solution $r(x)$ monotonically decreases from $4 m$ to $r_{1}$ on the interval $[0, p]$ and increases from $r_{1}$ to $r_{2}$ on $\left[p, t_{1}\right]$. After that it can oscillate between $r_{1}$ and $r_{2}$ several times and, at the end, $r(1 / 2)=r_{2}$ and $r(1)=4 m$. The function $r(x)$ cannot take a constant value on any subinterval of $I$ because of its analyticity.

I. The optimal shape of the strongest column is defined as

$$
Q_{0}(x)=\frac{r(x)^{2}}{9 m^{2}} .
$$

The values $k$ and $m$ can be found from the conditions

$$
\theta(1 / 2)-\theta(0)=-\pi, \quad r(1 / 2)=r_{2},
$$

where $\theta=\arctan (v-c) / u$, and

$$
\frac{d \theta}{d x}=-\frac{9 m^{2} c_{2}}{\rho\left(c_{1}-\rho\right)^{2}}=-\frac{9 m^{2} c_{2}}{r^{2}\left(c_{1}-r\right)} ;
$$

this means that

$$
\begin{gathered}
2 \int_{r_{1}}^{4 m} \frac{r^{2} d r}{\sqrt{P(r)}}+\int_{r_{2}}^{4 m} \frac{r^{2} d r}{\sqrt{P(r)}}=\sqrt{3 m} \\
2 \int_{r_{1}}^{4 m} \frac{d r}{\left(c_{1}-r\right) \sqrt{P(r)}}+\int_{4 m}^{r_{2}} \frac{d r}{\left(c_{1}-r\right) \sqrt{P(r)}}=\frac{2 \pi}{c_{2}(3 m)^{3 / 2}} .
\end{gathered}
$$

The last system of two equations for $k$ and $m$ can be solved numerically. The minimal value of $m$ corresponds to $p_{1}=2$ and $p_{2}=1$ and is equal to 0.01908 .

The integrals involved in this system are improper. In order to work with them, we use the factorization

$$
\begin{array}{cc}
P(r)=\left(r-r_{1}\right)\left(a_{0}+a_{1} r+a_{2} r^{2}-r^{3}\right), & r_{1}<r<4 m, \\
P(r)=\left(r_{2}-r\right)\left(b_{0}+b_{1} r+b_{2} r^{2}+r^{3}\right), & 4 m<r<r_{2},
\end{array}
$$


with

$$
a_{2}=c_{1}-r_{1}, \quad a_{1}=r_{1} a_{2}, \quad a_{0}=r_{1} a_{1}, \quad b_{2}=r_{2}-c_{1}, \quad b_{1}=r_{2} b_{2}, \quad b_{0}=r_{2} b_{1}
$$

and the substitution

$$
r=r_{1}+s^{2}, \quad r=r_{2}-t^{2}
$$

regularizing the integrals.

\section{Proof of Theorem 1}

A. Set

$$
L[Q, u, v]=\frac{\int_{0}^{1} Q(x)\left(u^{\prime}(x)^{2}+v^{\prime}(x)^{2}\right) d x}{\int_{0}^{1}\left(u(x)^{2}+v(x)^{2}\right) d x} .
$$

Let $u_{0}=u$ and $v_{0}=v$ be the functions found in Lemma 2 such that

$$
\int_{0}^{1}\left(u_{0}^{\prime}(x)^{2}+v_{0}^{\prime}(x)^{2}\right)^{-2} d x=1 .
$$

We set

$$
Q_{0}(x)=\left(u_{0}^{\prime}(x)^{2}+v_{0}^{\prime}(x)^{2}\right)^{-2} .
$$

We know that $Q_{0} \in C^{\infty}[0,1]$ and $Q_{0}(x)=Q_{0}(1-x)$.

Let us show that

$$
L\left[Q_{0}, u_{0}, v_{0}\right] \geq \sup _{Q \in \mathcal{A}} \inf _{u, v \in S \times S} L[Q, u, v] .
$$

We have

$$
L\left[Q_{0}, u_{0}, v_{0}\right]=\frac{\int_{0}^{1} Q_{0}(x)\left(u_{0}^{\prime}(x)^{2}+v_{0}^{\prime}(x)^{2}\right) d x}{\int_{0}^{1}\left(u_{0}(x)^{2}+v_{0}(x)^{2}\right) d x}=\frac{\int_{0}^{1}\left(u_{0}^{\prime}(x)^{2}+v_{0}^{\prime}(x)^{2}\right)^{-1} d x}{\int_{0}^{1}\left(u_{0}(x)^{2}+v_{0}(x)^{2}\right) d x}=\frac{1}{m},
$$

since

$$
\int_{0}^{1}\left(u_{0}^{\prime}(x)^{2}+v_{0}^{\prime}(x)^{2}\right)^{-1} d x=1 .
$$

On the other hand, if $Q$ is a positive function from $C^{1}(I)$ and

$$
\int_{0}^{1} Q^{1 / 2}(x) d x=1
$$

then, by Lemma 5 , we have $L[Q, u, v] \leq 1 / m$.

Therefore, for any $Q \in \mathcal{A}$, we have

$$
\inf _{\substack{u \in S \\ v \in S}} L[Q, u, v] \leq \inf _{\substack{u \in S_{0} \\ v \in S_{0}}} L[Q, u, v] \leq \frac{1}{m} \equiv M .
$$

Thus,

$$
\sup _{Q} \inf _{\substack{u \in S \\ v \in S}} L[Q, u, v] \leq M, \quad \sup _{Q} \inf _{y \in S} L_{1}[Q, y] \leq M
$$


B. Now we show that

$$
\inf _{y \in S} L_{1}\left[Q_{0}, y\right]=\inf _{\substack{u \in S \\ v \in S}} L\left[Q_{0}, u, v\right]=\inf _{\substack{u \in S_{1} \\ v \in S_{2}}} L\left[Q_{0}, u, v\right]=M
$$

By Lemma $2, u_{0} \not \equiv 0$ and $v_{0} \not \equiv 0$. Moreover, let

$$
\inf _{u \in S_{\text {odd }}} L\left[Q_{0}, u, 0\right]=\mu .
$$

This minimal value is attained for a function $u_{1}$ which is positive in ]0,1/2[. It satisfies the Lagrange equation

$$
\left(Q_{0} u_{1}^{\prime}(x)\right)^{\prime}+\mu u_{1}(x)=0
$$

$u_{1}(0)=0$, and $u_{1}(1 / 2)=0$.

The function $u_{0}$ is odd and positive in $] 0,1 / 2\left[\right.$, and $\left(Q_{0}(x) u_{0}^{\prime}(x)\right)^{\prime}+M u_{0}(x)=0$. Therefore,

$$
(M-\mu) \int_{0}^{1 / 2} u_{1}(x) u_{0}(x) d x=0 .
$$

The functions $u_{0}$ and $u_{1}$ are positive in $] 0,1 / 2\left[\right.$. Therefore, $M=\mu$ and $u_{1}=k u_{0}$.

C. Now let

$$
\inf _{v \in S_{\text {even }}} L\left[Q_{0}, 0, v\right]=\mu_{1} .
$$

This minimal value is attained for a function $v_{1}$ which is positive in $] 0, p[$ and negative in $] p, 1 / 2[$. It satisfies the Lagrange equation

$$
\left(Q_{0} v_{1}^{\prime}(x)\right)^{\prime}+\mu_{1} v_{1}(x)=C^{\prime}
$$

$v_{1}(0)=0$, and $v_{1}^{\prime}(1 / 2)=0$, where $C^{\prime}=-2 Q_{0}(0) v_{1}^{\prime}(0)<0$.

The function $v_{0}$ is positive in $] 0, p_{0}$ [ and negative in $] p_{0}, 1 / 2\left[\right.$, and $\left(Q_{0}(x) v_{0}^{\prime}(x)\right)^{\prime}+M v_{0}(x)=C$ with $C=-2 Q_{0}(0) v_{0}^{\prime}(0)<0$. Therefore, the functions $w_{0}=v_{0}-C / M$ and $w_{1}=v_{1}-C^{\prime} / \mu_{1}$ satisfy the equations

$$
\left(Q_{0} w_{0}^{\prime}\right)^{\prime}+M w_{0}=0, \quad\left(Q_{0} w_{1}^{\prime}\right)^{\prime}+\mu w_{1}=0
$$

Moreover,

$$
\begin{aligned}
& w_{0}(0)=-\frac{C}{M}, \quad M \int_{0}^{1 / 2} w_{0}(x) d x=Q_{0}(0) w_{0}^{\prime}(0), \quad \int_{0}^{1 / 2} w_{0}(x) d x=-\frac{C}{2 M}, \\
& w_{1}(0)=-\frac{C^{\prime}}{\mu_{1}}, \quad \mu_{1} \int_{0}^{1 / 2} w_{1}(x) d x=Q_{0}(0) w_{1}^{\prime}(0), \quad \int_{0}^{1 / 2} w_{1}(x) d x=-\frac{C^{\prime}}{2 \mu_{1}} .
\end{aligned}
$$

Therefore, they are eigenfunctions of the Sturm-Liouville operator corresponding to the boundary problem

$$
\left(Q_{0} z^{\prime}\right)^{\prime}+\lambda z=0, \quad \lambda z(0)=2 Q_{0}(0) z^{\prime}(0), \quad z^{\prime}(1 / 2)=0,
$$

considered in Lemma 4 . Since both $w_{0}$ and $w_{1}$ have exactly one zero in $[0,1 / 2]$, they are linearly dependent, i.e., $w_{1}(x)=k^{\prime} w_{0}(x)$. Therefore, $v_{1}(x)=k^{\prime} v_{0}(x)+C^{\prime \prime}$. But $v_{0}(0)=v_{1}(0)=0$; therefore, $C^{\prime \prime}=0$, $v_{1}=k^{\prime} v_{0}$, and $\mu_{1}=M$. 
D. If $y \in S$, then $y=u+v$, where $u \in S_{\text {odd }}$ and $v \in S_{\text {even. }}$ Therefore,

$$
L_{1}\left[Q_{0}, y\right]=\frac{\int_{0}^{1 / 2} Q_{0}(x)\left[u^{\prime}(x)^{2}+v^{\prime}(x)^{2}\right] d x}{\int_{0}^{1 / 2}\left[u(x)^{2}+v(x)^{2}\right] d x} \geq M
$$

since

$$
\int_{0}^{1 / 2} Q_{0}(x) u^{\prime}(x)^{2} d x \geq M \int_{0}^{1 / 2} u(x)^{2} d x, \quad \int_{0}^{1 / 2} Q_{0}(x) v^{\prime}(x)^{2} d x \geq M \int_{0}^{1 / 2} v(x)^{2} d x,
$$

as we have shown. We obtain

$$
\inf _{y \in S} L_{1}\left[Q_{0}, y\right]=\inf _{\substack{u \in S_{1} \\ v \in S_{2}}} L\left[Q_{0}, u, v\right]=L_{1}\left[Q_{0}, y_{0}\right]=M
$$

and $y_{0}=u_{0}+v_{0}$.

Acknowledgments. The author thanks A. P. Seyranian for useful consultations. This research was partially supported by the INTAS project INTAS-00-015.

\section{REFERENCES}

1. T. Clausen, "Über die form architektonischer Saulen," Acad. St-Petersbourg, IX (1851), pp. 371-380.

2. S. J. Cox, "The shape of the ideal column," Math. Intelligencer, 14, No. 1, 16-24 (1992).

3. S. J. Cox and M. L. Overton, "On the optimal design of columns against buckling," SIAM J. Math. Anal., 23, No. 2, 287-325 (1992).

4. Yu. V. Egorov and V. A. Kondratiev, "On the spectral theory of elliptic operators," in: Operator Theory, 89, Birkhäuser, Basel-Boston-Berlin (1996).

5. J. B. Keller, "The shape of the strongest column," Arch. Rat. Mech. Anal., 4, 275-285 (1960).

6. J. B. Keller and I. Tadjbakhsh, "Strongest columns and isoperimetric inequalities for eigenvalues," Trans. ASME, J. Appl. Mech., 29, No. 1, 159-164 (1962).

7. J.-L. Lagrange, "Sur la figure des colonnes," in: Euvres de Lagrange (Publ. de M. J.-A. Serret), Vol. 2, Gauthier-Villars, Paris (1868), pp. 125-170.

8. E. F. Masur, "Optimal structural design under multiple eigenvalue constraints," Int. J. Solids Struct., 20, No. 3, 211-231 (1984).

9. N. Olhoff and S. H. Rasmussen, "On single and bimodal optimum buckling loads of clamped columns," Int. J. Solids Struct., 13, No. 7, 605-614 (1977).

10. A. P. Seyranian, "A solution of a problem of Lagrange," Dokl. Akad. Nauk SSSR, 271, No. 2, 337-340 (1983).

11. A. P. Seyranian, "The Lagrange problem on optimal columns," Preprint 60-2000, Institute of Mechanics, Moscow State Univ. (2000).

12. A. P. Seyranian, "On a Lagrange problem," Izv. Akad. Nauk SSSR, Mekh. Tverd. Tela, 2, 101-111 (1984).

Yu. V. Egorov

Paul Sabatier University, UFR MIG, Labo MIP, UMR 5640

E-mail: egorov@mip.ups-tlse.fr 